\title{
Nuclear Localization of the Serine/Threonine Kinase DRAK2 Is Involved in UV-Induced Apoptosis
}

\author{
Hiroshi Kuwahara, Norihiro Nakamura, and Hiroshi Kanazawa* \\ Department of Biological Sciences, Graduate School of Science, Osaka University; 1-1 Machikaneyama-cho, Toyonaka, \\ Osaka 560-0043, Japan. Received October 15, 2005; accepted November 21, 2005; published online November 28, 2005
}

\begin{abstract}
DAP kinase-related apoptosis-inducing kinase 2 (DRAK2), a member of the DAP kinase family, is a serine/threonine kinase capable of inducing apoptosis. Here we studied the relationship between DRAK2 intracellular localization and apoptosis, and found that UV light acts as a stimulus for apoptosis induced by DRAK2. The intracellular location of DRAK2 depended on the cell line: DRAK2 was found primarily in the nuclei of NRK, NIH3T3, and Caco-2 cells while it was present primarily in the cytoplasm of ACL-15, HeLa, and WI-38 cells. Overexpression of Myc-tagged DRAK2 led to apoptosis-like cell death in NRK cells, but not in ACL-15 cells. A GFP fusion protein of DRAK2 was spontaneously localized to the nucleus of ACL-15 cells and resulted in cell death. Nuclear localization and cell death were also observed with DRAK2(1-293) fused to the NLS of SV40 but not with DRAK2(1-293) alone. These results suggested that nuclear accumulation of DRAK2 and the resulting increase in the endogenous level of its kinase activity are required for cell death. UV irradiation caused nuclear accumulation of endogenous DRAK2 in ACL-15 cells, which was followed by apoptosis-like cell death. Knockdown of DRAK2 expression by siRNA partially suppressed UV-induced apoptosis. These results suggest that DRAK2 plays a role in UV induced apoptosis.
\end{abstract}

Key words apoptosis; nuclear localization; DAP kinase-related apoptosis-inducing kinase 2

Apoptosis, a type of programmed cell death, plays an essential role in the survival of multicellular organisms and is observed in a wide variety of physiological situations. ${ }^{1)}$ Apoptosis is important for biological self-defense, and defects in the ability to undergo apoptosis can lead to various diseases, including cancer, neurodegenerative diseases, and autoimmune diseases. ${ }^{2}$ ) Apoptosis is a well-defined cellular process characterized by morphological changes that include condensation of nuclear chromatin, cell shrinkage, and plasma membrane blebbing. ${ }^{3)}$

The Fas family cytokine and tumor necrosis factor (TNF) cascades can mediate apoptosis, ${ }^{4,5)}$ but recently, many additional apoptotic components have been identified. Using a functional cloning strategy, Deiss et al. identified the deathassociated protein kinase (DAPK) as a positive mediator of apoptosis triggered by interferon- $\gamma \cdot{ }^{6}$ ) The kinase activity of DAPK, which shares homology with myosin light chain kinases, is regulated by calmodulin in a $\mathrm{Ca}^{2+}$-dependent manner in vitro. ${ }^{7)}$ Overexpression of DAPK in various cell lines results in cell death, and this death-promoting property strictly depends on the intrinsic kinase activity. Additional isoforms of the DAPK family, including DRP-1, ZIP kinase, DAP kinase-related apoptosis-inducing kinase 1 (DRAK1), and DRAK2 have been identified. These proteins share homology in their primary structures, especially in the catalytic domain. ${ }^{8-11)}$ All of these kinases have an apoptosis-promoting activity that is dependent on the kinase activity.

We have studied the function of the small $\mathrm{Ca}^{2+}$-binding protein CHP1 (calcineurin B homologous protein1). ${ }^{12)} \mathrm{CHP1}$ is a multifunctional regulator of various proteins, including $\mathrm{Na}^{+} / \mathrm{H}^{+}$antiporters, a kinesin-like motor protein, and calcineurin. ${ }^{13-15)}$ We identified DRAK2 as a CHP1 binding protein, and found that CHP1 inhibits the kinase activity of DRAK2 in a $\mathrm{Ca}^{2+}$-dependent manner. ${ }^{16)}$ DRAK2 is found in thymus, testis, spleen, and brain, tissues in which apoptosis plays an important role. ${ }^{12)}$ Overexpression of DRAK2 in NIH3T3 cells causes apoptosis like cell death through a mechanism that requires the kinase activity. ${ }^{10)}$ However, overexpression studies do not necessarily indicate whether DRAK2 causes apoptosis during normal physiological processes.

Several components of apoptotic signal transduction pathways, such as DNases, Bcl family members, and caspases, are localized to specific areas of the cell. ${ }^{17)}$ During UV-induced apoptosis, damaged double stranded DNA signals translocation of the mitochondrial components AIF and Endo-G to the nucleus. ${ }^{18,19)} \mathrm{UV}$ irradiation also activates cytoplasmic targets, such as the transcriptional factors AP-1 and $\mathrm{NF}-\kappa \mathrm{B} .{ }^{20,21)} \mathrm{NF}-\kappa \mathrm{B}$ migrates to the nucleus and binds to specific response elements of various genes. Thus, the intracellular localization and translocation of molecules are important elements of apoptosis, and understanding these dynamics will elucidate the regulatory mechanisms of apoptosis.

The DAPK family members ZIP kinase and DAP kinase are localized in the nucleus and cytoplasm, respectively. ${ }^{7,22}$ ZIP kinase is located within promyelocytic leukemia oncogenic domains (PODs) and induces apoptosis in response to apoptotic stimuli such as $\mathrm{As}_{2} \mathrm{O}_{3}$ or interferon- $\gamma^{23)}$ Although we have previously shown that DRAK2 is localized in the nucleus in COS-7 cells ${ }^{12)}$ and causes apoptosis like cell death in NIH3T3 cells, the correlation between cell death and the intracellular localization of DRAK2 has not been systematically studied. In this study, we examined the correlation between the intracellular localization of DRAK2 and cell death to identify the putative signal transduction pathways involved in apoptotic cell death. We found that the translocation of DRAK is cell type dependent; in certain types of cells, DRAK is spontaneously accumulated in the nucleus, while in other types of cells, it is mainly detected in the cytoplasm. In the case of the former types of cells, ectopically overexpressed DRAK2 induces cell death. In latter types of cells, UV-irradiation causes nuclear translocation of endogenous DRAK2 followed by apoptotic cell death. 


\section{MATERIALS AND METHODS}

Cell Lines and Culture Conditions ACL-15 cells derived from rat colon cancer were cultured in RPMI containing $10 \%$ fetal calf serum, $2 \mathrm{~mm}$ L-glutamine, $1 \mathrm{~mm}$ sodium pyruvate, and 100 units $/ \mathrm{ml}$ penicillin. Normal rat kidney cells (NRK), mouse fibroblasts (NIH3T3), simian kidney cells (COS-7), and human cervical carcinoma cells (HeLa) were cultured in Dulbecco's modified Eagle medium (DMEM) with 10\% fetal calf serum, $2 \mathrm{~mm}$ L-glutamine, $1 \mathrm{~mm}$ sodium pyruvate, and 100 units $/ \mathrm{ml}$ penicillin. Human colon carcinoma cells (Caco-2) and normal human fibroblasts (WI38) were cultured in Eagle's minimal essential medium with $10 \%$ or $20 \%$ fetal calf serum, $2 \mathrm{~mm}$ L-glutamine, $1 \mathrm{~mm}$ sodium pyruvate, and 100 units $/ \mathrm{ml}$ penicillin. Media and reagents required for cell culture were obtained from SigmaAldrich (St. Louis, MO, U.S.A.) and Invitrogen (Carlsbad, CA, U.S.A.). Cells were incubated in a humidified atmosphere of $5 \% \mathrm{CO}_{2}$ and $95 \%$ air at $37^{\circ} \mathrm{C}$. For transfection of plasmid DNA, cells were incubated with a mixture of $\mathrm{Fu}$ GENE 6 (Roche Diagnostics, Basel, Switzerland) and DNA, according to the manufacturer's instructions.

Antibodies Monoclonal antibodies against $\beta$-tubulin (clone DM1A) and c-Myc (clone 9E10) were purchased from Sigma-Aldrich (St. Louis, MO, U.S.A.) and Santa Cruz Biotechnology (Santa Cruz, CA, U.S.A.), respectively. The polyclonal antibody against Oct-1 was purchased from Santa Cruz Biotechnology (Santa Cruz, CA, U.S.A.). Secondary antibodies conjugated to Alexa Fluor 488 and Alexa Fluor 546 were purchased from Molecular Probes (Eugene, OR, U.S.A.). Affinity purified anti-DRAK2 antibodies raised against the C-terminal portion of DRAK2 (residues 227371) have been described previously. ${ }^{12)}$

Expression Plasmids The plasmid encoding Myctagged DRAK2 was described previously. ${ }^{12)}$ To express Myctagged DRAK2(1-293) fused to the SV40 NLS, oligonucleotides corresponding to the SV40 NLS sequence (PKKKRKV) with recognition sequences for BamHI at both $5^{\prime}$ - and $3^{\prime}$-ends were synthesized. After BamH1 digestion, the fragment was inserted in-frame into the $B a m \mathrm{H} 1$ site between the Myc-tag and DRAK2(1-293) in pEF-BOS-EX. Expression vectors encoding Myc-DRAK2 were digested with $\operatorname{BamHI}$, and then the digested fragment was inserted into the pEGFP-C3 plasmid (Clontech, Palo Alto, CA, U.S.A.) to create the GFP-DRAK2 expression vector.

Immunofluorescence Microscopy Coverslips were coated with poly-L-lysine (Sigma \#P-1399, Sigma-Aldrich, St. Louis, MO, U.S.A.). Cells seeded onto coated coverslips were fixed for 25 min with phosphate buffered saline (PBS) containing $2 \%$ formaldehyde, and the residual formaldehyde was then quenched using $0.1 \mathrm{M}$ glycine in PBS. ${ }^{24)}$ After incubation in PBS containing $0.4 \%$ saponin, $1 \%$ bovine serum albumin, and $2 \%$ goat serum, the samples were incubated with the primary antibodies for $1 \mathrm{~h}$, followed by incubation with secondary antibodies for $1 \mathrm{~h}$. The samples were covered with $50 \%$ glycerol in PBS, placed on slides, and then observed using a BX-51 microscope equipped with a 40x UplanApo objective (Olympus, Melville, NY, U.S.A.). The fluorescence of the Alexa Fluor 488- and Alexa Fluor 546-conjugated secondary antibodies, Hoechst 33342, and GFP was observed with excitation filters of $470-490 \mathrm{~nm}, 520-550 \mathrm{~nm}, 330$
$385 \mathrm{~nm}$, and $460-490 \mathrm{~nm}$ and emission filters of 515$555 \mathrm{~nm}, 580 \mathrm{~nm}, 420 \mathrm{~nm}$, and $510 \mathrm{~nm}$, respectively. Digital images were acquired with an ORCA-ER1394 digital camera and then processed with AQUA-COSMOS software (Hamamatsu Photonics, Hamamatsu, Japan).

Subcellular Fractionation and Immunoblotting ACL15 and NRK cells cultured in $100-\mathrm{mm}$ culture dishes were washed with $2 \mathrm{ml}$ PBS, scraped in PBS, and collected by centrifugation $(1000 \times \boldsymbol{g}$ for $5 \mathrm{~min})$. ACL-15 cells were washed in $1 \mathrm{ml}$ of cell-lysis buffer (10 mM Tris- $\mathrm{HCl}$ [pH 7.5], $10 \mathrm{~mm} \mathrm{NaCl}$, and $1 \mathrm{~mm}$ phenylmethylsulfonyl fluoride). After centrifugation at $1000 \times \boldsymbol{g}$ for $5 \mathrm{~min}$, the cells in the pellet were resuspended in $200 \mu \mathrm{l}$ of cell-lysis buffer and then homogenized by 30 passages through a 26 -gauge needle. ${ }^{25)}$ NRK cells were washed in $1 \mathrm{ml}$ of cell-lysis buffer and centrifuged $(1000 \times \mathbf{g}$ for $5 \mathrm{~min})$, and the cell pellet was resuspended in $300 \mu \mathrm{l}$ of cell lysis buffer. Homogenization was performed using a Dounce homogenizer. The cell lysate was centrifuged $(1000 \times \boldsymbol{g}$ for $5 \mathrm{~min}$ ), and the pellet (nuclei) and supernatant (cytoplasm) were collected. To eliminate DNA aggregates, both the cytoplasmic and nuclear enriched fractions were sonicated. The fractionated proteins were solubilized in SDS-polyacrylamide gel electrophoresis (SDSPAGE) sample buffer. The samples were separated by SDSPAGE and transferred to PVDF membranes (Millipore, Billerica, MA, U.S.A.). The membranes were incubated with the indicated primary antibodies and then with secondary horseradish peroxidase-conjugated antibodies against rabbit or mouse IgG (Jackson ImmunoResearch Laboratories, West Grove, PA, or Vector Laboratories, Burlingame, CA, U.S.A.). The signals were detected with ECL-plus or ECL Western Blotting Detection Reagent and Hyperfilm-ECL (Amersham Biosciences, Piscataway, NJ, U.S.A.), as described previously. ${ }^{24)}$

UV Irradiation For microscopic observation, approximately $1 \times 10^{5}$ cells were plated in $35-\mathrm{mm}$ cell culture dishes $48 \mathrm{~h}$ before UV irradiation and maintained in a complete medium (RPMI-1640 medium containing 10\% FBS). The medium was removed, and the cells were irradiated at $50 \mathrm{~J} / \mathrm{m}^{2}$ by a germicidal lamp with peak emission at $254 \mathrm{~nm}$ (CL-1000, UVP, Inc., Upland, CA, U.S.A.). Immediately after UV irradiation, the cells were maintained in the same medium at $37^{\circ} \mathrm{C}$ in $5 \% \mathrm{CO}_{2}$ for the indicated times.

Phase Contrast Microscopy Cells plated in 35-mm culture dishes were transfected with the indicated plasmid vectors. Forty-eight hours after transfection, the cells were labeled for $5 \mathrm{~min}$ at $37^{\circ} \mathrm{C}$ in $2 \mathrm{ml}$ of RPMI- 1640 medium containing $1 \mu \mathrm{M}$ Hoechst 33342 and were observed using an IX70 phase contrast microscope with a 40x LCPlanFl objective (Olympus, Melville, NY, U.S.A.). GFP and Hoechst fluorescence were observed with excitation filters of $460-490 \mathrm{~nm}$ and $330-385 \mathrm{~nm}$ and emission filters of $510 \mathrm{~nm}$ and $420 \mathrm{~nm}$, respectively.

Preparation and Transfection of siRNAs For siRNA experiments, double-stranded RNA duplexes composed of 25-nucleotide sense and antisense oligoribonucleotides were synthesized by Invitrogen (Carlsbad, CA, U.S.A.). These siRNAs were chemically modified, and efficient knockdown of target mRNA was achieved. The RNA oligonucleotide used to target DRAK2 was 5'-AACAAAUCUGG AACGAGUUCGUGGG-3'. This sequence did not show signifi- 
cant homology to any other genes by BLAST search (NCBI). A control siRNA, which showed no homology to any genes, was purchased from Invitrogen. ACL-15 or NRK cells at $50 \%$ confluence in 12-well plates were transfected with 250 pmol of each of the above siRNAs using Lipofectamine 2000 (Invitrogen, Carlsbad, CA, U.S.A.), according to the manufacturer's protocol. Transfected cells were incubated at $37^{\circ} \mathrm{C}$ for $24 \mathrm{~h}$. The effect of the siRNA on the expression of DRAK2 was measured by Western blot analysis using an anti-DRAK2 antibody.

\section{RESULTS}

Intracellular Localization of DRAK2 in Various Cell Lines We determined the subcellular localization of DRAK2 in several cell lines. As reported previously, ${ }^{12}$ ) DRAK2 was localized in the nucleus of COS-7 cells. When Myc-tagged DRAK2 was transiently expressed in NRK (rat), NIH3T3 (mouse), and Caco-2 (human) cells, DRAK2 was also found primarily in the nucleus (Fig. 1A). In NRK cells, the distribution of endogenous DRAK2 and that of transiently expressed Myc-tagged DRAK2 were not distinguishable (Fig. 1C). In contrast to these results, Myc-tagged DRAK2 was observed primarily in the cytoplasm in ACL-15 (rat), HeLa (human), and WI-38 (human) cells (Figs. 1B, C). In ACL-15 cells, endogenous DRAK2 was also localized to the cytoplasm. To confirm the distribution of endogenous DRAK2, we fractionated ACL-15 and NRK cells into nuclei and cytoplasm (Fig. 1D). Since our antibody against DRAK2 reacts only with rat DRAK2, endogenous DRAK2 of human and mouse cells could not be observed. Consistent with the immunostaining results, DRAK2 in ACL-15 cells was predominantly detected in cytoplasm, whereas it was detected in nuclei in NRK cells. The total amount of DRAK2 in ACL-15 was lower than that in NRK. Preliminary observations of rat tissues showed that endogenous DRAK2 is found in the nuclei of thymus cells and in the cytoplasm of cerebellar Purkinje cells (Kanazawa et al., unpublished observations), supporting the previous observations that the location of DRAK2 differs by cell type.

The Carboxy-Terminal Domain and the Kinase Activity of DRAK2 Are Required for Nuclear Accumulation A mutant DRAK2, DRAK2(1-293), that still possesses the kinase domain and kinase activity but contains a truncation in the C-terminus, is unable to induce apoptosis in NIH3T3 cells. ${ }^{10)}$ This result was unexpected because the kinase activity required for the induction of apoptosis was still present in the mutant protein. We therefore examined the subcellular localization of DRAK2(1-293). We found that MycDRAK2(1-293) was distributed in the cytoplasm of NRK and NIH3T3 cells (Figs. 2A, D), indicating that the C-terminal domain plays a role in the nuclear translocation of
A

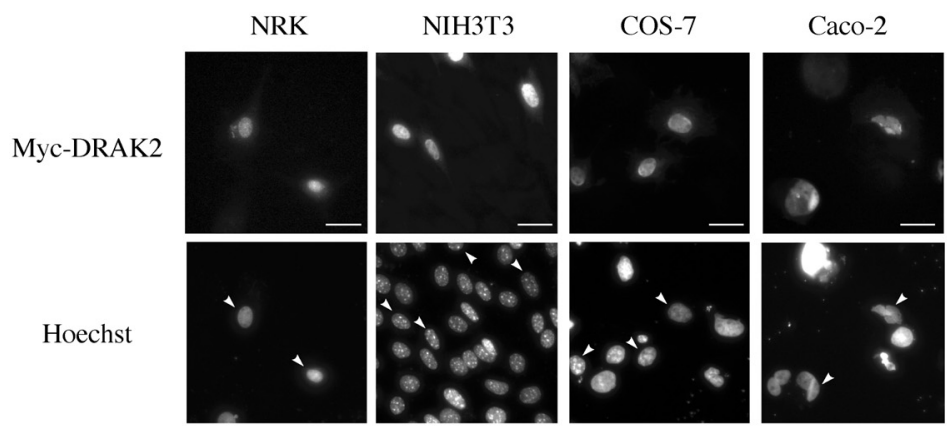

B

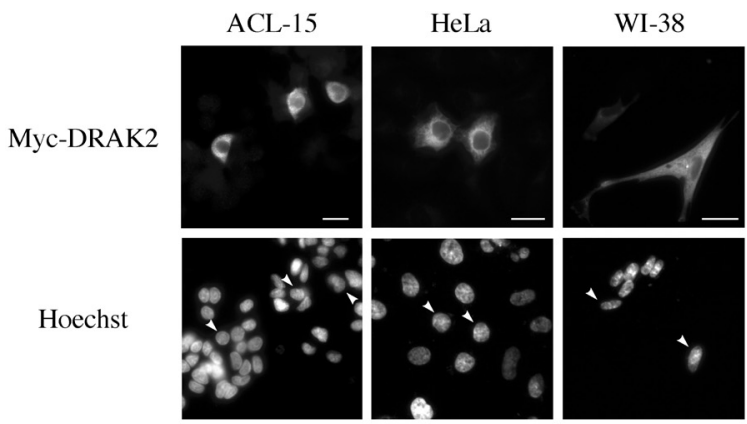

C

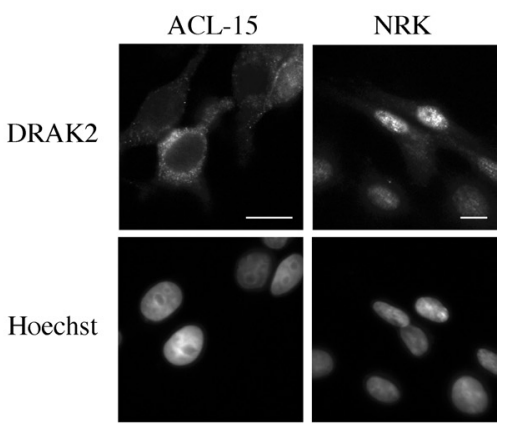

$\mathrm{D}$

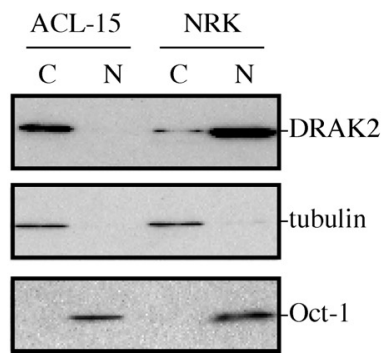

Fig. 1. Localization of DRAK2 in Various Cell Lines

(A) NRK, NIH3T3, COS-7, and CaCo-2 cells. (B) ACL-15, HeLa, and WI-38 cells. Cells were transfected with an expression vector encoding Myc-DRAK2, and the distribution of DRAK2 was visualized $48 \mathrm{~h}$ post-transfection by indirect immunofluorescence using a Myc-tag antibody and an Alexa Fluor 546-conjugated secondary antibody. Nuclei were stained with Hoechst 33342. The arrowhead indicates the transfected cells. Bar, $20 \mu \mathrm{m}$. (C) ACL-15 and NRK cells were fixed, permeabilized, and stained with an antiDRAK2 rabbit polyclonal antibody followed by an Alexa Fluor 488-conjugated anti-rabbit IgG. Nuclei were stained with Hoechst 33342. Bar, $20 \mu \mathrm{m}$. (D) Nuclear (N) and cytoplasmic (C) fractions were prepared from ACL-15 or NRK cells, as described in the Materials and Methods, and analyzed by immunoblotting using antibodies against DRAK2 (top panel). Immunoblots of cytoplasmic and nuclear extracts were also probed with antibodies against $\beta$-tubulin (cytoplasmic protein marker) or Oct-1 (nuclear protein marker) (middle and bottom panel). 
A

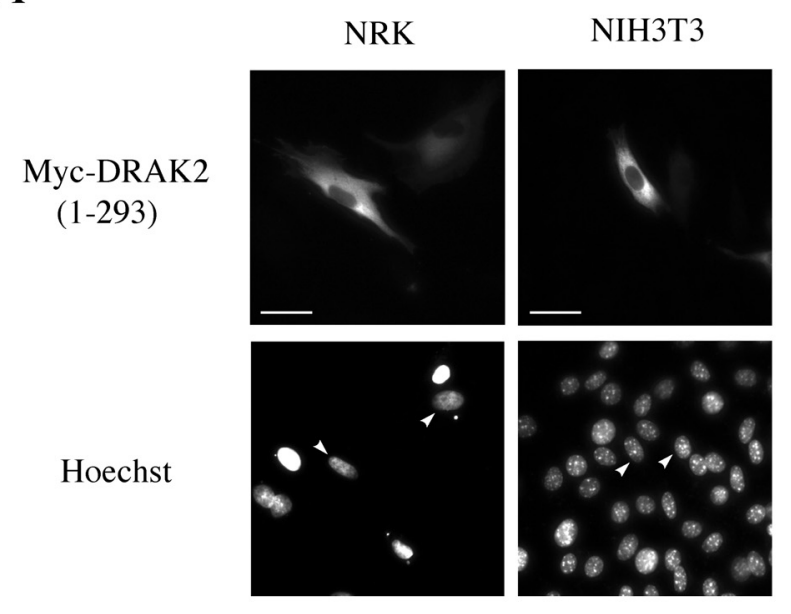

B

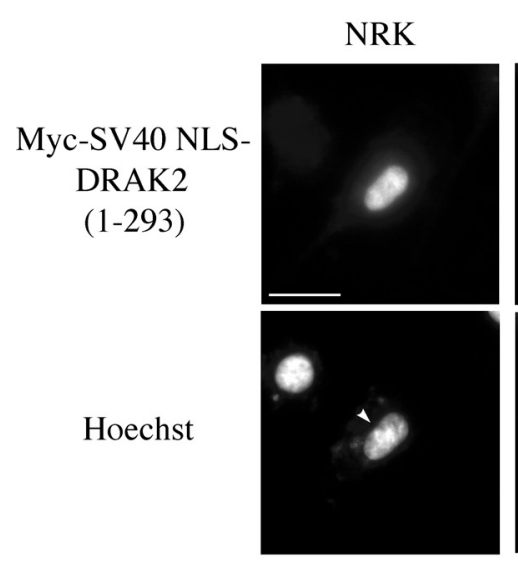

NIH3T3

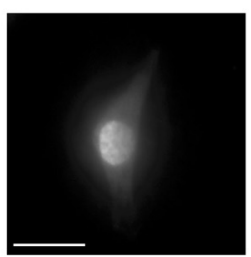

C

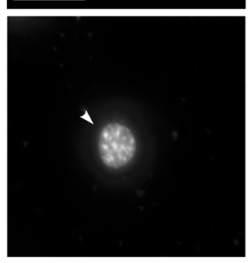

K62A

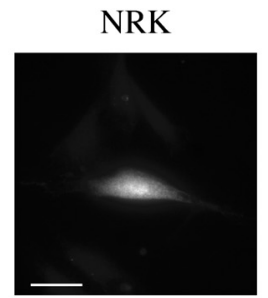

NIH3T3

Hoechst
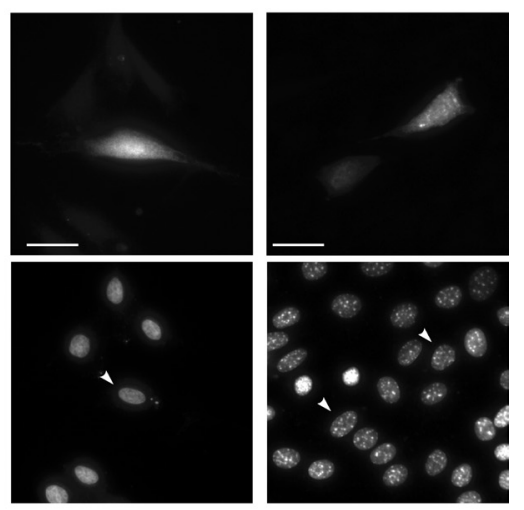

D

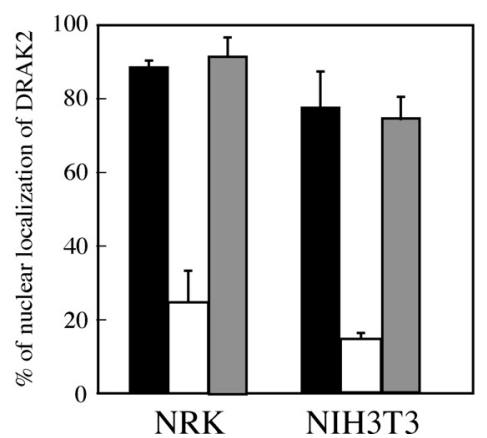

Fig. 2. Localization of DRAK2(1-293), a Mutant with a C-Terminal Truncation

(A) NRK and NIH3T3 cells were transfected with an expression vector encoding the C-terminally truncated mutant Myc-DRAK2(1-293), and the distribution was visualized $48 \mathrm{~h}$ post-transfection using a Myc-tag antibody and an Alexa Fluor 546-conjugated secondary antibody. The nuclei were shown by staining with Hoechst 33342 . The arrowhead indicates transfected cells. Bar, $20 \mu \mathrm{m}$. (B) A heterologous NLS from SV40 was inserted between the Myc-tag and the DRAK2 coding region and the construct was transfected into NRK and NIH3T3 cells. The distribution pattern of the Myc-SV40 NLS-DRAK2(1-293) was examined $48 \mathrm{~h}$ post-transfection by indirect immunofluorescence microscopy. (C) As in (B), the kinase inactive mutant DRAK2(K62A) was overexpressed in NRK and NIH3T3 cells and $48 \mathrm{~h}$ later its distribution pattern was observed. (D) Myc-DRAK2 constructs (wild-type, 1-293, or SV40 NLS +1 - 293) were overexpressed in NRK or NIH3T3 cells, and 48 h later distribution of DRAK2 was observed. The percentages of cells showing DRAK2 nuclear localization are shown. One hundred percent corresponds to the total number of analyzed cells transfected by Myc-DRAK2. Filled, open, and gray bars represent wild-type-, 1-293-, and SV40 NLS+1-293 transfected cells, respectively. At least 100 transfected cells were analyzed in each of three independent experiments (mean \pm standard deviation).

DRAK2. To confirm this observation, we fused the SV40 nuclear localization signal (NLS) to DRAK2(1-293) and examined its cellular localization. As shown in Figs. 2B and D,
DRAK2(1-293) fused to the NLS of SV40 was localized to the nucleus, indicating that the C-terminal region (294-371) of DRAK2 is required for its nuclear translocation. Next, we 
A

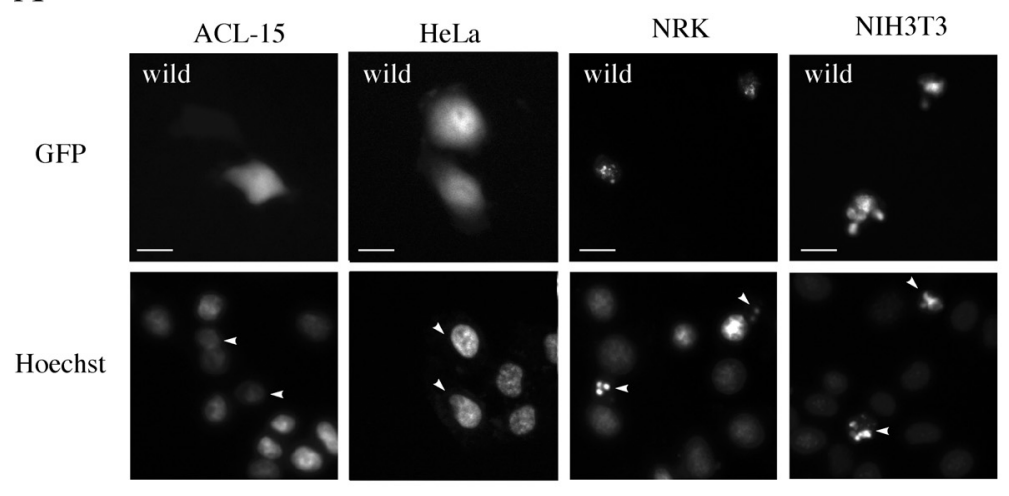

B

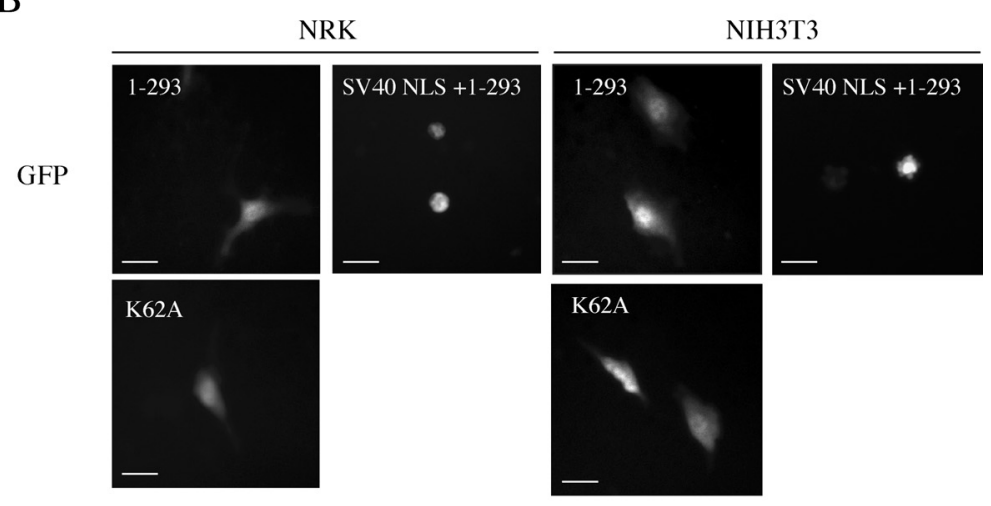

$\mathrm{C}$

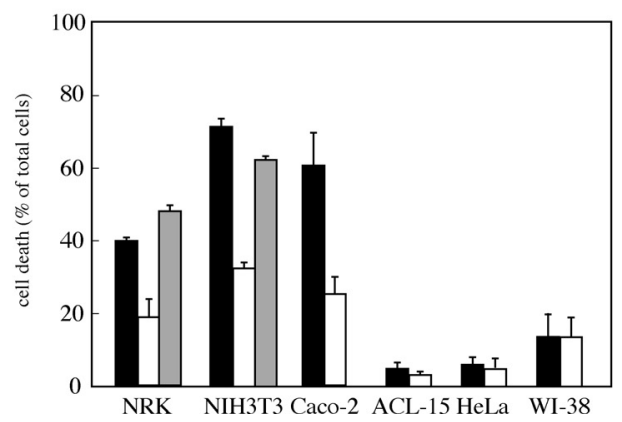

Fig. 3. Cell Death Induced by Overexpression of DRAK2 in Various Cultured Cell Lines

(A) Cells were co-transfected with plasmids encoding GFP (1 ng) or Myc-DRAK2 $(500 \mathrm{ng})$. At $48 \mathrm{~h}$ post-transfection, the nuclei were stained with Hoechst 33342 , and GFPpositive cells were visualized with fluorescence microscopy. Expression of Myc-DRAK2 in GFP-positive cells were confirmed by immunostaining with Myc-tag antibodies. GFPexpressing cells are indicated by arrows. Bar, $20 \mu \mathrm{m}$. (B) NRK and NIH3T3 cells were co-transfected with the plasmids encoding GFP (1 ng) or the C-terminally truncated MycDRAK2 (1-293) fused to the SV40 NLS, or the kinase inactive DRAK2(K62A) (500 ng). GFP-positive cells were visualized with fluorescence microscopy $48 \mathrm{~h}$ after transfection Bar, $20 \mu \mathrm{m}$. (C) Cells were co-transfected with the plasmids encoding GFP (1 ng) or Myc-DRAK2 (500 ng) (wild-type, 1-293, or 1-293 with SV40 NLS). At $48 \mathrm{~h}$ post-transfection, GFP-positive cells were stained with Hoechst 33342 and the numbers of apoptotic cells were scored by their nuclear morphology under fluorescence microscopy. The ratio is shown as a percentage of GFP-positive cells. Filled, open, and gray bars represent wild-type, 1-293, and 1-293 with SV40 NLS DRAK2 transfected cells, respectively. The data represent the average numbers of three independent experiments and at least $100 \mathrm{GFP}$ positive cells were analyzed in each experiment (mean \pm standard deviation).

tested whether the kinase activity of DRAK2 is required for its nuclear translocation. In contrast to wild type DRAK2, the Myc-tagged kinase-inactive DRAK2(K62A) was scattered throughout the cell and did not accumulate in the nucleus (Fig. 2C). This result suggested that the kinase activity in addition of the $\mathrm{C}$-terminal domain is required for nuclear accumulation of DRAK2.

Accumulation of DRAK2 in the Nucleus, But Not in the Cytoplasm, Causes Cell Death Because MycDRAK2(1-293) was found primarily in the cytoplasm, we hypothesized that the nuclear localization of DRAK2 might be required to induce cell death. In ACL-15 cells, the distribution of DRAK2 is restricted mainly to the cytoplasm and we tested whether overexpression of DRAK2 in these cells could induce cell death. When Myc-tagged DRAK2 was introduced into ACL-15 cells, only a very small fraction of the transfected cells underwent cell death (Figs. 3A, C). Similar results were observed with HeLa and WI-38 cells. In contrast, overexpression of Myc-DRAK2 in NRK, NIH3T3, and Caco-2 cells induced morphological alterations similar to those seen in apoptosis (Figs. 3A, C). Overexpression of DRAK2(1-293) did not cause significant morphological changes in any of these cell lines (Figs. 3B, C). However, addition of the SV40 NLS to DRAK2(1-293) caused translocation of DRAK2 to the nucleus (Figs. 2B, D) and induced cell death (Figs. 3B, C). The kinase inactive mutant, 
A
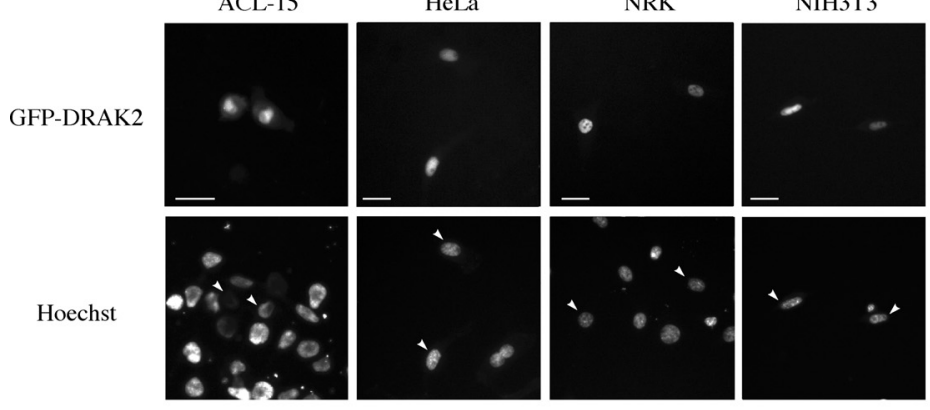

B
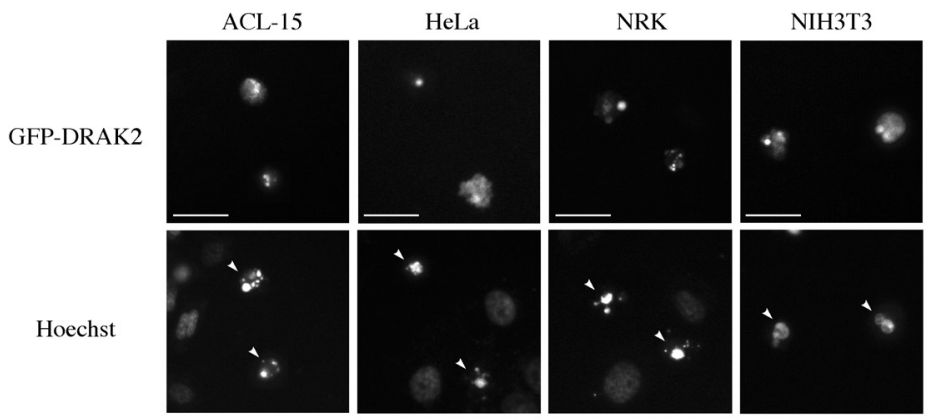

C

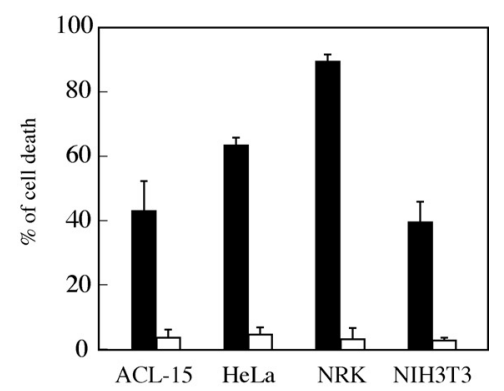

D

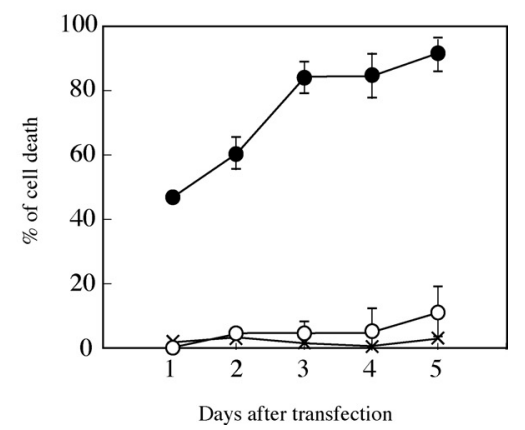

Fig. 4. Expression of GFP-DRAK2 Fusion Protein in ACL-15, HeLa, NRK, and NIH3T3 Cells

Cells were transfected with plasmids encoding GFP-DRAK2. The cells were stained with Hoechst 33342 and viewed with a fluorescence microscope. Localization of DRAK2 was visualized by direct fluorescence of EGFP. The arrowhead indicates the transfected cells. Bar, $20 \mu$ m. (A) Twenty-four hours after transfection. (B) Forty-eight hours after transfection. (C) Cells were transfected with GFP-DRAK2 or GFP expression plasmids. After $48 \mathrm{~h}$, cells with typical apoptotic morphology, including membrane blebbing, were counted. Filled and open bars represent GFP-DRAK2-transfected and GFP-transfected cells, respectively. The fraction of apoptotic cells is expressed as a percentage of GFP-positive cells. At least 300 cells were counted (mean \pm standard deviation; $n=3$ ). (D) ACL-15 cells were transfected with a plasmid carrying GFP, GFP-DRAK2, or a combination of GFP (1 ng) and Myc-DRAK2 (500 ng). One to $5 \mathrm{~d}$ after transfection, GFP-positive cells were examined using fluorescence microscopy, and the percentage of GFP-positive cells showing apoptotic morphology was determined. Closed circles $(\bullet)$ are GFP-DRAK2-transfected cells, open circles $(\bigcirc)$ represent Myc-DRAK2-transfected cells, and $(\times)$ are GFP-transfected cells. The fraction of apoptotic cells is expressed as a percentage of GFP-positive cells. At least 150 GFP-positive cells were counted (mean \pm standard deviation; $n=3)$.

DRAK2(K62A), did not induce cell death, although there was significant increase in the nuclear level of DRAK2(K62A) compared to that of intact DRAK2 (Figs. $2 \mathrm{C}, 3 \mathrm{~B}$, and data not shown). These results strongly suggested that when nuclear expression of DRAK2 is increased above the endogenous level, cell death is induced and DRAK2-associated kinase activity is required for this response.

To confirm the localization of DRAK2 in living cells, we introduced GFP-DRAK2 expression plasmids into the cells. The kinase activity of the fusion protein was equivalent to that of the original DRAK2 (data not shown). The GFP fluorescence was found primarily in the nucleus in NRK and NIH3T3 cells (Fig. 4A) and cell death occurred, as expected (Fig. 4B). However, GFP-DRAK2 was found in the nucleus as well as the cytoplasm of ACL-15 and HeLa cells (Fig. $4 \mathrm{~A}$ ), and the proportion of death cells increased concomitantly (Figs. 4C, D), suggesting that the nuclear localization is sufficient for cell death in ACL-15 and HeLa cells.

Endogenous DRAK2 Translocates to the Nucleus after UV Irradiation We investigated the conditions that affect the nuclear accumulation of endogenous DRAK2 and subsequent cell death. UV irradiation stimulated both nuclear accumulation of DRAK2 and apoptosis in ACL-15 cells. Membrane blebbing was observed after exposure to UV, and the number of apoptotic cells increased with longer incubation times after irradiation (Fig. 5A).

To understand the role of DRAK2 in UV-induced apoptosis, we used a DRAK2 antibody to analyze the subcellular localization of DRAK2 by indirect immunofluorescence. In ACL-15 cells, DRAK2 was observed throughout the cytoplasm and was clearly distinguishable from the nucleus (Fig. 5B). Cytoplasmic DRAK2 decreased by $4 \mathrm{~h}$ after exposure to UV light, while nuclear DRAK2 increased, suggesting that DRAK2 had translocated into the nucleus. Similar results were observed at $24 \mathrm{~h}$ after UV irradiation. Other apoptotic stimuli like 5-fluorouracil and cisplatin induce apoptosis to ACL-15 cells, but these drugs did not cause translocation of DRAK2 like UV-irradiation (Fig. 6). The population of cells expressing DRAK2 in the nucleus increased rapidly after UV irradiation (Fig. 5C) and the total amount of protein was unchanged during nuclear translocation of DRAK2 (Fig. 5D). These results imply that nuclear translocation of DRAK2 occurs prior to apoptosis.

siRNA-Mediated Knockdown of Endogenous DRAK2 Protects Cells against UV-Induced Apoptosis To examine whether DRAK2 is required for UV-induced apoptosis, we generated siRNA targeted to DRAK2. The DRAK2-specific siRNA was transfected into ACL-15 and NRK cells, and a siRNA that did not show homology to any genes in the vertebrate transcriptome was used as a control. Cell lysates were prepared $24 \mathrm{~h}$ after transfection and immunoblot analysis 


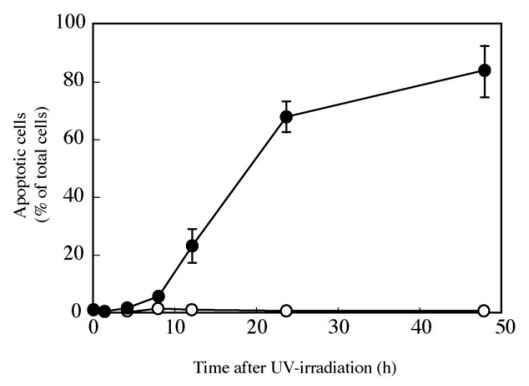

B

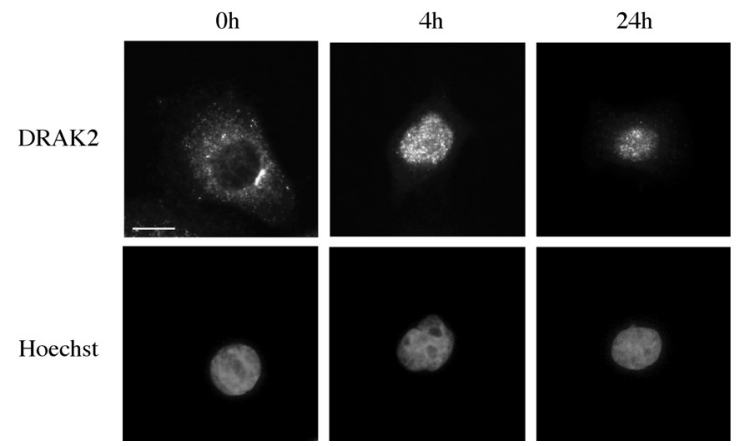

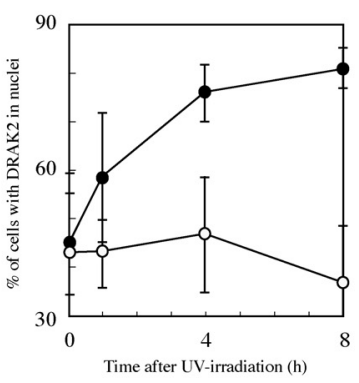

D

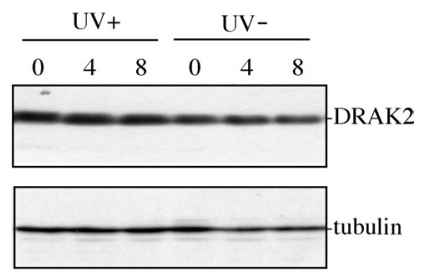

Fig. 5. Translocation of Endogenous DRAK2 to the Nucleus after UV Irradiation of ACL-15 Cells

(A) ACL-15 cells were UV irradiated and stained with acridine orange and ethidium bromide $(0.1 \mathrm{mg} / \mathrm{ml}$ each). Apoptotic cells were scored at $0,1,4,12,24$, and $48 \mathrm{~h}$ after UVirradiation based on standard morphological hallmarks, including membrane blebbing. At least 300 fluorescent cells were analyzed, and data are expressed as a percentage of the total number analyzed. Closed $(\bullet)$ and open $(\mathrm{O})$ circles represent UV-irradiated and non-irradiated cells, respectively (mean \pm standard deviation; $n=3$ ). (B) ACL-15 cells cultured on coverslips coated with poly-L-lysine were exposed to UV light (wavelength $254 \mathrm{~nm}$ ) at $50 \mathrm{~J} / \mathrm{m}^{2}$ for $3 \mathrm{~s}$. Cells were then fixed at 0,4 , and $24 \mathrm{~h}$ after irradiation and stained with anti-DRAK2 antibodies and Hoechst 33342. DRAK2 was detected using an Alexa Fluor 488-conjugated anti-rabbit IgG followed by fluorescence microscopy. Bar, $10 \mu \mathrm{m}$. (C) The number of cells exhibiting predominantly nuclear localization of DRAK2 at the indicated time after UV irradiation is presented as a percentage of all of the cells scored. At least 300 cells were analyzed. Closed $(\bullet)$ and open $(\bigcirc)$ circles represent UV-irradiated and non-irradiated cells, respectively (mean \pm standard deviation; $n=3$ ). (D) Cell lysates of UV-irradiated or non-irradiated ACL-15 cells were prepared at 0,4 , or $8 \mathrm{~h}$ after irradiation, and analyzed by immunoblot for DRAK2 (top panel) and the cytoplasmic marker $\beta$-tubulin (bottom panel).

A

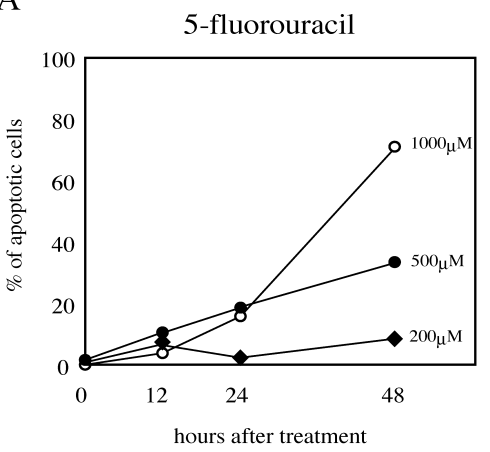

cisplatin

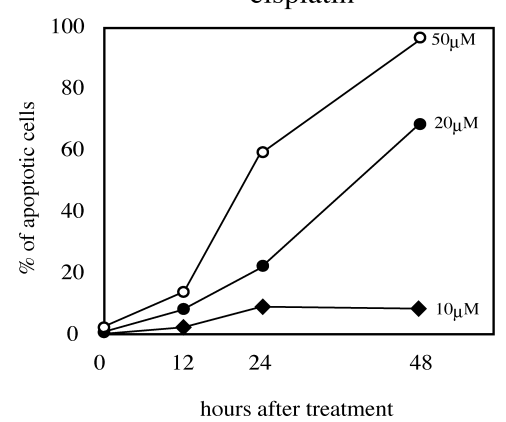

$\mathrm{B}$

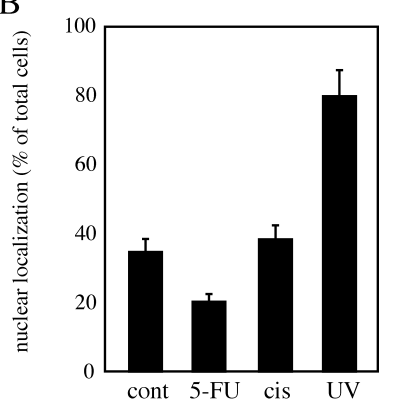

Fig. 6. Effect of 5-Fluorouracil and Cisplatin on the Localization of DRAK2 in ACL-15

(A) ACL-15 cells were incubated with 5 -fluorouracil $(200-1000 \mu \mathrm{M})$ or cisplatin $(10-50 \mu \mathrm{M})$, and numbers of the apoptotic cells were scored at 0 , 12,24 and $48 \mathrm{~h}$ after addition of the drugs. At least 300 fluorescent cells were analyzed. (B) Cells were incubated with $1000 \mu \mathrm{M} 5$-fluorouracil (5-FU) or $20 \mu \mathrm{M}$ cisplatin (cis) for $24 \mathrm{~h}$ and then numbers of cells exhibiting predominantly nuclear localization of DRAK2 were counted (mean \pm standard deviation; $n=3$ ). Percentages of cells exhibiting nuclear localization after UV-irradiation (UV) and the control cells without irradiation (cont) are also presented.

confirmed that targeted siRNA transfection achieved a ca. $80 \%$ reduction in DRAK2 protein level, but had no effect on the expression of a control protein, $\beta$-tubulin (Fig. 7A). DRAK2 expression was suppressed for up to $3 \mathrm{~d}$, as determined by immunoblot analysis (data not shown).

DRAK2 knockdown cells began to show apoptotic morphology $12 \mathrm{~h}$ after UV irradiation. For both the ACL-15 and
NRK cells, $20-30 \%$ of the cells transfected with control siRNA or mock-treated cells showed apoptotic cell death by $12 \mathrm{~h}$ after exposure to UV light (Fig. 7B). In contrast, the knockdown of DRAK2 expression caused a significant reduction in the number of cells undergoing cell death (Fig. 7B). These results suggested that DRAK2 expression plays a role in UV induced apoptosis. 
A

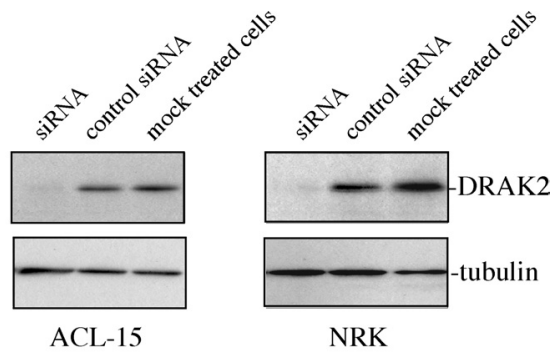

B

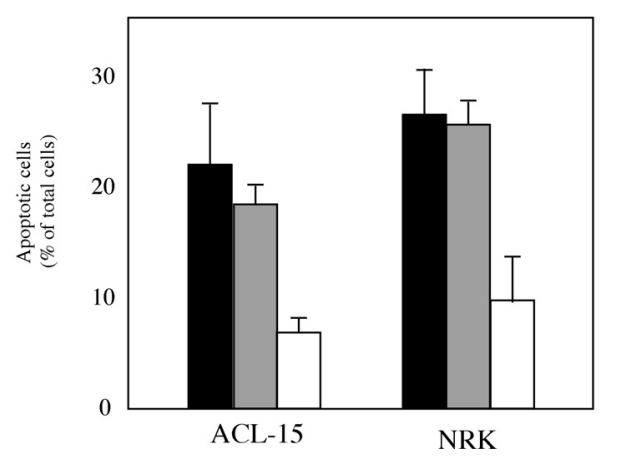

Fig. 7. Involvement of DRAK2 in UV-Induced Apoptosis as Evidenced by RNA Interference

(A) An siRNA construct was transfected into ACL-15 cells and NRK cells. Cells transfected with control siRNA or mock treated were also prepared. Twenty-four hours after transfection, cells were harvested and lysed in $\operatorname{PBS}(-)$ and analyzed by immunoblot for DRAK2 (top panel) or $\beta$-tubulin (bottom panel). (B) NRK and ACL-15 cells, with or without endogenous DRAK2, were UV irradiated, and $12 \mathrm{~h}$ later apoptotic cells were scored based on the morphological changes. At least 300 fluorescent cells were analyzed, and the data are expressed as a percentage of the total number analyzed. Filled, gray, and open bars represent mock treated, control siRNA transfected, and specific siRNA transfected cells, respectively (mean \pm standard deviation; $n=3$ ).

\section{DISCUSSION}

In this study we found that DRAK2 localizes to the nucleus or the cytoplasm, depending on the cell line. In ACL15 , HeLa, and WI-38 cells, DRAK2 is primarily cytoplasmic, whereas it is located in the nucleus in NRK, NIH3T3, COS-7, and Caco-2 cells. However, when truncated DRAK2 (1-293) was overexpressed, it was observed in the cytoplasm of all cell types examined. The kinase inactive DRAK2(K62A) was scattered throughout the cell, but did not accumulate in the nucleus. UV irradiation or GFP fusion caused DRAK2 to translocate into the nucleus in ACL-15 cells. These results suggest that DRAK2 might transiently be present in the nucleus and cytoplasm of all cells, although the final localization of DRAK2 differs among cell lines. This difference might be determined by a putative factor that binds to DRAK2 and alters its cellular localization. The expression of this putative factor or the state of autophosphorylation of the C-terminal region of DRAK2 might differ between cell lines. These hypotheses are consistent with the localizations of truncated DRAK2(1-293) and the kinase-inactive form of DRAK2. Unexpectedly, the GFP-DRAK2 fusion localized to the nucleus. The fusion might cause a conformational change in DRAK2 that facilitates nuclear translocation. Alternatively, the GFP-DRAK2 conformation might decrease the binding of an accessory factor, allowing DRAK2 to translocate into the nucleus via a putative NLS (nuclear localization signal) in the C-terminal region. We have obtained a preliminary result that suggests presence of NLS in the C-terminal region between residues 297 and 371. Further studies of DRAK2 localization in these cell lines will be required to elucidate the molecular mechanism of its nuclear localization.

Overexpression of DRAK2 in the cytoplasm of ACL-15 cells did not cause cell death. However, nuclear localization of the GFP-DRAK2 fusion in ACL-15 cells did stimulate cell death. While DRAK2(1-293), which was restricted to the cytoplasm, did not induce cell death despite its high kinase activity, the overexpression of this form with an SV40 NLS did lead to accumulation in the nucleus and cell death. UV exposure induced translocation of DRAK2 from the cytoplasm to the nucleus and caused apoptosis. These independent lines of evidence strongly support the hypothesis that both the accumulation of DRAK2 in the nucleus and the kinase activity are required to induce cell death. While endogenous DRAK2 does not cause cell death in NRK and NIH3T3 cells, cell death was induced when exogenous DRAK2 was expressed in these cells. This result suggested that the endogenous expression level of DRAK2 is insufficient to induce cell death and that an increase in the expression of DRAK2 may be required for these cells. The putative targets of DRAK2 kinase activity are most likely present in the nucleus. Nuclear accumulation of DRAK2 might be required because the endogenous level of DRAK2 in the nucleus in these cell lines is below the level required for the interaction with its putative target. Another possibility might be that overexpression is required to overcome a putative masking or inhibitory factor of DRAK2 activity.

This is the first study to identify UV irradiation as an apoptotic stimulus involving endogenous DRAK2. The translocation of DRAK2 into the nucleus in response to UV irradiation occurred prior to apoptosis, and UV-induced apoptotic cell death was partially inhibited by knockdown of DRAK2. Several proteins are translocated into the nucleus when apoptosis is induced, ${ }^{26)}$ and many of them have DNase or protease activities and work as effector molecules in the execution of apoptosis. ${ }^{27-29)}$ Based on its primary structure, DRAK2 is unlikely to have these activities, but may instead work as a mediator that transfers apoptotic signals into the nucleus, rather than working as an effector. UV irradiation is known to break double-stranded DNA and activates signaling molecules such as NF- $\kappa$ B and JNK ${ }^{30-32)}$ Like c-Abl, which is indirectly regulated by JNK, DRAK2 might transfer apoptotic signals into the nucleus. ${ }^{33)} \mathrm{A}$ factor in the nucleus, probably the target of DRAK2 phosphorylation, may then be involved in transducing the apoptotic signal of DRAK2. Since RNAi-mediated knockdown of DRAK2 only partially suppressed UV-induced apoptosis, it is likely that pathways other than those involving DRAK2 exist.

While DAPK and DRP-1 were thought to be cytoplasmic proteins, ZIP kinase, DRAK1, and DRAK2 were thought to be nuclear proteins. ${ }^{34)}$ These differences in intracellular localization of DAPK family members may be due to divergence of regions outside the kinase domain. The mechanism DRAK2 uses to induce apoptosis is different from that seen with other DAPK family members, especially DAPK and DRP-1. ${ }^{35,36)}$ However, ZIPK is known to localize to PML bodies in the nucleus. ${ }^{23)}$ This process is induced by IFN and ZIPK phosphorylates Par-4, leading to apoptosis. Histone 
phosphorylation by ZIPK has also been reported. ${ }^{37)}$ Thus, putative targets of DRAK2 kinase activity could be Par- 4 and histones, similar to ZIPK. However, it has also been argued that cytoplasmic localization of ZIPK is required for apoptosis. ${ }^{38)}$ Thus, the precise mechanism of apoptosis induced by DAPK family members including DRAK2 will require further study.

Recently, McGargill et al. reported that DRAK2 deficient mouse established by them did not reveal obvious growth defect. ${ }^{39)}$ They also showed that $\mathrm{T}$ cells derived from the deficient mouse was highly sensitive to T-cell stimuli but did not show any defect in T-cell apoptosis. We and other group reported that DRAK2 is able to cause cell death for NIH3T3 and other cell lines shown here. Moreover, DRAK1, an isoform of DRAK2 has been shown to be involved in apoptotic process of osteoclast. ${ }^{40)}$ Therefore, physiological function of DRAK2 in terms of apoptosis not only for immune system but also other tissues should require further studies including fine inspection of the DRAK2 deficient mouse.

Acknowledgements The present study was supported by a Grant-in-Aid for a scientific research from the Japanese Ministry of Science, Education, Culture, Sports and Technology. The authors thank Dr. Hiroki Inoue and Jun-ichi Kamei for helpful discussions throughout this study.

\section{REFERENCES}

1) Smith C. A., Williams G. T., Kingston R., Jenkinson E. J., Owen J. J., Nature (London), 338, 10 (1989).

2) Barisic K., Petrik J., Rumora L., Acta Pharm., 53, 151-164 (2003).

3) Wyllie A. H., Kerr J. F., Currie A. R., Int. Rev. Cytol., 68, 251-306 (1980).

4) Nagata S., Annu. Rev. Genet., 33, 29-55 (1999).

5) Gaur U., Aggarwal B. B., Biochem. Pharmacol., 66, 1403-1408 (2003).

6) Deiss L. P., Feinstein E., Berissi H., Cohen O., Kimchi A., Genes Dev., 9, 15-30 (1995)

7) Cohen O., Feinstein E., Kimchi A., EMBO J., 16, 998-1008 (1997).

8) Kawai T., Matsumoto M., Takeda K., Sanjo H., Akira S., Mol. Cell. Biol., 18, 1642-1651 (1998).

9) Kogel D., Plottner O., Landsberg G., Christian S., Scheidtmann K. H., Oncogene, 17, 2645-2654 (1998).

10) Sanjo H., Kawai T., Akira S., J. Biol. Chem., 273, 29066-29071 (1998).

11) Inbal B., Shani G., Cohen O., Kissil J. L., Kimchi A., Mol. Cell. Biol., 20, 1044-1054 (2000)

12) Matsumoto M., Miyake Y., Nagita M., Inoue H., Shitakubo D., Takemoto K., Ohtsuka C., Murakami H., Nakamura N., Kanazawa H., J. Biochem. (Tokyo), 130, 1044-1054 (2001).

13) Lin X., Sikkink R. A., Rusnak F., Barber D. L., J. Biol. Chem., 274, 36125-36131 (1999).
14) Pang T., Su X., Wakabayashi S., Shigekawa M., J. Biol. Chem., 276, 17367-17372 (2001).

15) Nakamura N., Miyake Y., Matsushita M., Tanaka S., Inoue H., Kanazawa H., J. Biochem. (Tokyo), 132, 483-491 (2002).

16) Kuwahara H., Kamei J., Nakamura N., Matsumoto M., Inoue H., Kanazawa H., J. Biochem. (Tokyo), 134, 245-250 (2003).

17) Gross A., McDonnell J. M., Korsmeyer S. J., Genes Dev., 13, 1899 1911 (1999).

18) Susin S. A., Lorenzo H. K., Zamzami N., Marzo I., Snow B. E., Brothers G. M., Mangion J., Jacotot E., Costantini P., Loeffler M., Larochette N., Goodlett D. R., Aebersold R., Siderovski D. P., Penninger J. M., Kroemer G., Nature (London), 397, 441-446 (1999).

19) Arnoult D., Gaume B., Karbowski M., Sharpe J. C., Cecconi F., Youle R. J., EMBO J., 22, 4385-4399 (2003).

20) Devary Y., Gottlieb R. A., Lau L. F., Karin M., Mol. Cell. Biol., 11, 2804-2811 (1991).

21) Devary Y., Rosette C., DiDonato J. A., Karin M., Science, 261, 14421445 (1993).

22) Page G., Kogel D., Rangnekar V., Scheidtmann K. H., Oncogene, 18 , 7265-7273 (1999).

23) Kawai T., Akira S., Reed J. C., Mol. Cell. Biol., 23, 6174-6186 (2003).

24) Matsushita M., Tanaka S., Nakamura N., Inoue H., Kanazawa H., Traffic, 5, 140-151 (2004).

25) Nakamura N., Yamamoto A., Wada Y., Futai M., J. Biol. Chem., 275, $6523-6529$ (2000).

26) Yasuhara N., Eguchi Y., Tachibana T., Imamoto N., Yoneda Y., Tsujimoto Y., Genes Cells, 2, 55-56 (1997).

27) Enari M., Sakahira H., Yokoyama H., Okawa K., Iwamatsu A., Nagata S., Nature (London), 391, 43-50 (1998).

28) Mao P. L., Jiang Y., Wee B. Y., Porter A. G., J. Biol. Chem., 273, 23621-23624 (1998).

29) Sahara S., Aoto M., Eguchi Y., Inamoto N., Yoneda Y., Tujimoto Y., Nature (London), 401, 168-173 (1999).

30) Aragane Y., Kulms D., Luger T. A., Schwarz T., Proc. Natl. Acad. Sci. U.S.A., 94, 11490-11495 (1997).

31) Tournier C., Hess P., Yang D. D., Xu J., Turner T. K., Nimnual A., BarSagi D., Jones S. N., Flavell R. A., Davis R. J., Science, 288, 870-874 (2000).

32) Sinha R. P., Hader D. P., Photochem. Photobiol. Sci., 1, 225-236 (2002).

33) Yoshida K., Yamaguchi T., Natsume T., Kufe D., Miki Y., Nat. Cell Biol., 3, 278-285 (2005).

34) Shohat G., Shani G., Eisenstein M., Kimchi A., Biochim. Biophys. Acta, 1600, 45-50 (2002).

35) Raveh T., Berissi H., Eisenstein M., Spivak T., Kimchi A., Proc. Natl. Acad. Sci. U.S.A., 97, 1572-1577 (2000).

36) Kogel D., Prehn J. H., Scheidtmann K. H., Bioessays, 23, 352-358 (2001).

37) Preuss U., Landsberg G., Scheidtmann K. H., Nucleic Acids Res., 31, 878 -885 (2002)

38) Kogel D., Bierbaum H., Preuss U., Scheidtmann K. H., Oncogene, 18, 7212-7218 (1999).

39) McGargill M. A., Wen B. G., Walsh C. M., Hedrick S. M., Immunity, 6, 781-791 (2004).

40) Kojima H., Nemoto A., Uemura T., Honma R., Ogura M., Liu Y., J. Biol. Chem., 276, 19238-19243 (2001). 